\title{
Effect of quality, porosity and density on the compression properties of cork
}

Anjos O., Pereira H., Rosa M.E., 2008. Holz als Roh-und Werkstoff, 66 (4): 295-301.

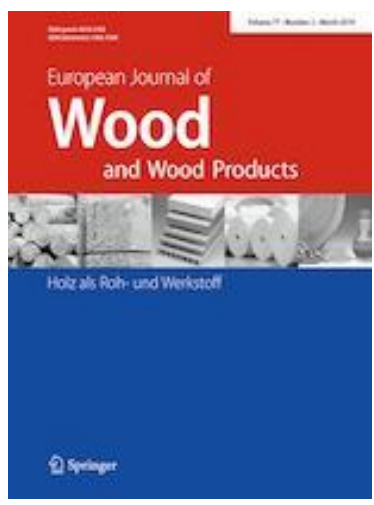

https://link.springer.com/article/10.1007/s00107-008-0248-2

\begin{abstract}
The compression properties of cork were studied on samples obtained from cork planks of two commercial quality classes (good and poor quality), with densities ranging from $0.12-0.20 \mathrm{~g} \mathrm{~cm}^{-3}$ and porosities from 0.5 to $22.0 \%$. The stress-strain curves were characterized by an elastic region up to approximately $5 \%$ strain, followed by a large plateau up to $60 \%$ strain caused by the progressive buckling of cell walls, and a steep stress increase for higher strains corresponding to cell collapse. The direction of compression was a highly significant factor of variation, with cork showing higher strength for the radial compression.

Density influenced compression and cork samples with higher density showed overall larger resistance to compression in the three directions. In the elastic region, an exponential model of Young's modulus in function of cork density could be adjusted.

The effect of porosity on compression was small and the stress-strain curves were similar regardless of the porosity of the samples, although there was a trend toward an overall increase of stress with porosity for higher strains. Porosity was characterised by a high variability in the anatomical features of the lenticular filling material and the presence of collapsed and thick walled lignified cells. The inclusion of a porosity parameter for the modelling of the elastic modulus did not improve the prediction obtained with density-based models.

There was no significant difference in the compression properties of cork samples obtained from cork planks of good and poor quality classes.
\end{abstract}

Keywords: Quality Classis; Radial Compression; Cork Stopper; Cork Sample; Quality Cork 